Article

\title{
Minimum Time for the Evolution to a Nonorthogonal Quantum State and Upper Bound of the Geometric Efficiency of Quantum Evolutions
}

\author{
Carlo Cafaro $^{1, *}$ and Paul M. Alsing ${ }^{2}$ \\ 1 Department of Mathematics and Physics, SUNY Polytechnic Institute, Albany, NY 12203, USA \\ 2 Air Force Research Laboratory, Information Directorate, Rome, NY 13441, USA; paul.alsing@us.af.mil \\ * Correspondence: carlocafaro2000@yahoo.it
}

Citation: Cafaro, C.; Alsing, P.M.

Minimum Time for the Evolution to a

Nonorthogonal Quantum State and

Upper Bound of the Geometric

Efficiency of Quantum Evolutions.

Quantum Rep. 2021, 3, 444-457.

https: / / doi.org/10.3390/

quantum3030029

Academic Editor: Lev Vaidman

Received: 18 June 2021

Accepted: 12 August 2021

Published: 16 August 2021

Publisher's Note: MDPI stays neutral with regard to jurisdictional claims in published maps and institutional affiliations.

\begin{abstract}
We present a simple proof of the fact that the minimum time $T_{A B}$ for quantum evolution between two arbitrary states $|A\rangle$ and $|B\rangle$ equals $T_{A B}=\hbar \cos ^{-1}[|\langle A \mid B\rangle|] / \Delta E$ with $\Delta E$ being the constant energy uncertainty of the system. This proof is performed in the absence of any geometrical arguments. Then, being in the geometric framework of quantum evolutions based upon the geometry of the projective Hilbert space, we discuss the roles played by either minimum-time or maximumenergy uncertainty concepts in defining a geometric efficiency measure $\varepsilon$ of quantum evolutions between two arbitrary quantum states. Finally, we provide a quantitative justification of the validity of the inequality $\varepsilon \leq 1$ even when the system only passes through nonorthogonal quantum states.
\end{abstract}

Keywords: quantum computation; quantum information; quantum mechanics

\section{Introduction}

Several works have studied the minimum time for the quantum evolution to an orthogonal state. A first list of works on a lower bound on the orthogonality time based on the energy spread includes the investigations by Mandelstam and Tamm [1], Fleming [2], Anandan and Aharonov [3], and Vaidman [4]. In a second type of explorations, there are works such as the one by Margolous and Levitin [5] that express a lower bound on the orthogonalization time based on the average energy of the system. In Ref. [6], instead, Levitin and Toffoli proposed a lower bound that involves both the energy spread and the average energy of the system. These lower bounds were extended from isolated to non-isolated systems [7] and moreover, from pure to mixed quantum states in the presence of entanglement [8,9] as well. For a recent expository review of the minimum evolution time and quantum speed limit inequalities that includes generalizations to mixed system states and open multiparticle systems, we refer to Ref. [10] and the references therein.

It is known that the minimum time $T$ for the Schrödinger evolution to an orthogonal state for a system with a time-independent energy uncertainty $\Delta E$ is given by $T=h /(4 \Delta E)$ with $h$ denoting the Planck constant. This finding was proved, for example, by Vaidman in Ref. [4] without using any geometric argument, while it was proved by Anandan and Aharonov in Ref. [3] with the help of more elaborate geometric considerations within the framework of the projective Hilbert space geometry. In both Refs. [3,4], the expression for the minimum time was restricted to evolutions to an orthogonal quantum state. More specifically, Anandan and Aharonov derived a rather interesting inequality relating the time interval $\Delta t$ of the quantum evolution to the time-averaged uncertainty in energy $\langle\Delta E\rangle$ (during the time interval $\Delta t$ ) in Ref. [3]:

$$
\langle\Delta E\rangle \Delta t \geq h / 4 \text {. }
$$

In particular, they stated that the equality sign in Equation (1) holds if and only if the system moves along a geodesic in the projective Hilbert space. To quantify geodesic motion 
in the projective Hilbert space, they also introduced a notion of efficiency denoted as $\varepsilon$ (this symbol $\varepsilon$ will be replaced with $\eta_{\mathrm{QM}}^{\text {(geometric) }}$ in this manuscript), with $\varepsilon \leq 1$ containing the inequality in Equation (1) as a special case. However, they stated without proof that $\varepsilon \leq 1$ is generally valid even when the system does not pass through orthogonal states.

In this paper, inspired by the results presented in Refs. [3,4], we find the expression for the minimum time for the evolution to an arbitrary nonorthogonal quantum state. Moreover, based on this first result, we provide a quantitative justification of the validity of the inequality $\varepsilon \leq 1$ even when the system only passes through nonorthogonal states.

The layout of the paper is as follows. In Section 2, we present a derivation of the expression for the minimum time for the evolution to an arbitrary nonorthogonal quantum state. In Section 3, exploiting the result obtained in Section 2, we provide a quantitative justification of the validity of the inequality $\varepsilon \leq 1$ even when the system only passes through nonorthogonal states. In Section 4, we discuss in an explicit manner the concepts of minimum evolution time and quantum geometric efficiency in two physical examples. Finally, our final considerations appear in Section 5.

\section{Minimum Time without Geometric Arguments}

In this section, we provide proof that the minimum time $T_{A B}$ for the quantum evolution between two arbitrary states $|A\rangle$ and $|B\rangle$ is equal to $T_{A B}=\hbar \cos ^{-1}[|\langle A \mid B\rangle|] / \Delta E$ where $\Delta E$ denotes the constant energy uncertainty of the system. This proof closely follows the proof presented by Vaidman in Ref. [4] where, however, the quantum evolution was restricted to an orthogonal quantum state.

We begin with some preliminary remarks. Consider an operator $\hat{Q}$ and a normalized quantum state $|\psi\rangle$. The state $\hat{Q}|\psi\rangle$ can be decomposed as

$$
\hat{Q}|\psi\rangle=\alpha_{1}|\psi\rangle+\alpha_{2}\left|\psi_{\perp}\right\rangle,
$$

where $\alpha_{1}, \alpha_{2} \in \mathbb{C}$ and $|\psi\rangle \perp\left|\psi_{\perp}\right\rangle$. Then, we obtain:

$$
\begin{aligned}
\hat{Q}|\psi\rangle & =\alpha_{1}|\psi\rangle+\alpha_{2}\left|\psi_{\perp}\right\rangle \\
& =\left|\alpha_{1}\right| e^{i \varphi_{1}}|\psi\rangle+\left|\alpha_{2}\right| e^{i \varphi_{2}}\left|\psi_{\perp}\right\rangle \\
& =e^{i \varphi_{2}}\left[\left|\alpha_{1}\right| e^{i\left(\varphi_{1}-\varphi_{2}\right)}|\psi\rangle+\left|\alpha_{2}\right|\left|\psi_{\perp}\right\rangle\right] .
\end{aligned}
$$

The state $\hat{Q}|\psi\rangle$ in Equation (3) is physically equivalent to the state $\left|\alpha_{1}\right| e^{-i \varphi}|\psi\rangle+$ $\left|\alpha_{2}\right|\left|\psi_{\perp}\right\rangle$ with $\varphi \stackrel{\text { def }}{=} \varphi_{2}-\varphi_{1}$ given that global phases have no relevance in quantum mechanics. Therefore, we conclude from Equation (3) that we can write the state $\hat{Q}|\psi\rangle$ as

$$
\hat{Q}|\psi\rangle=\alpha|\psi\rangle+\beta\left|\psi_{\perp}\right\rangle,
$$

with $\alpha \in \mathbb{C}$ and $\beta \in \mathbb{R}_{+}$. If we assume that $\hat{Q}$ is also Hermitian, we have:

$$
\alpha=\langle\psi|\hat{Q}| \psi\rangle=\left\langle\psi\left|\hat{Q}^{\dagger}\right| \psi\right\rangle=\langle\psi|\hat{Q}| \psi\rangle^{*}=\alpha^{*},
$$

that is, $\alpha \in \mathbb{R}$. Moreover, as it remains in the working condition with $\hat{Q}$ being Hermitian, the dispersion $\Delta Q^{2}$ of the operator $\hat{Q}$ becomes:

$$
\begin{aligned}
\Delta Q^{2} & =\left\langle\psi\left|\hat{Q}^{2}\right| \psi\right\rangle-\langle\psi|\hat{Q}| \psi\rangle^{2} \\
& =\left\langle\psi\left|\hat{Q} \hat{Q}^{\dagger}\right| \psi\right\rangle-\alpha^{2} \\
& =|\alpha|^{2}+|\beta|^{2}-\alpha^{2} \\
& =\alpha^{2}+\beta^{2}-\alpha^{2} \\
& =\beta^{2},
\end{aligned}
$$


that is:

$$
\beta=\Delta Q \text {. }
$$

Finally, using Equations (5) and (7), $\hat{Q}|\psi\rangle$ in Equation (4) can be decomposed as

$$
\hat{Q}|\psi\rangle=\langle\hat{Q}\rangle|\psi\rangle+\Delta Q\left|\psi_{\perp}\right\rangle,
$$

with $\left\langle\psi \mid \psi_{\perp}\right\rangle=0$. For an alternative derivation of Equation (8), we refer to Appendix A. Equation (8) will play a key role in our derivation of the minimum time expression.

At this point, we state the problem we wish to address. We want to find an explicit expression of the minimum time for the evolution from the normalized state $|A\rangle=|\psi(0)\rangle$ to the state $|B\rangle=\left|\psi\left(T_{A B}\right)\right\rangle$ with $\langle A \mid B\rangle \neq 0$ in the working condition that the dispersion of the Hamiltonian operator $\hat{\mathrm{H}}$ is constant:

$$
\Delta E=\sqrt{\left\langle A\left|\hat{\mathrm{H}}^{2}\right| A\right\rangle-\langle A|\hat{\mathrm{H}}| A\rangle^{2}}=\text { constant. }
$$

Before continuing our proof, we would like to emphasize at this point that our demonstration works equally well for time-dependent Hamiltonians $\hat{\mathrm{H}}(t)$ with non-constant energy uncertainty $\Delta E(t)$ where $\Delta E^{2}(t) \stackrel{\text { def }}{=}\left\langle\psi(t)\left|\hat{\mathrm{H}}^{2}\right| \psi(t)\right\rangle-\langle\psi(t)|\hat{\mathrm{H}}| \psi(t)\rangle^{2}$ and $|\psi(t)\rangle$ is assumed to be normalized to one. However, as we shall see, an expression of the minimum evolution time can only be obtained in an implicit manner when taking into consideration systems specified by this type of Hamiltonian.

Returning to our proof, we point out for the sake of completeness that since stationary states are quantum states with no energy uncertainty (that is, $\Delta E=0$ [11]), we shall limit our considerations to the quantum evolutions of nonstationary states [2]. Following the work by Vaidman $[4,12]$, the minimum time for the evolution to a nonorthogonal state can be found by evaluating the maximum of the absolute value of the rate of change of the modulus squared of the quantum overlap $\langle\psi(t) \mid A\rangle$ with $|\psi(t)\rangle$ being an intermediate state between $|A\rangle$ and $|B\rangle$ satisfying the time-dependent Schrödinger equation:

$$
i \hbar \partial_{t}|\psi(t)\rangle=\hat{\mathrm{H}}|\psi(t)\rangle .
$$

Let us consider the quantity $d\left[|\langle\psi(t) \mid A\rangle|^{2}\right] / d t$. Observe that:

$$
|\langle\psi(t) \mid A\rangle|^{2}=\langle\psi(t) \mid A\rangle\langle\psi(t) \mid A\rangle^{*}=\langle\psi(t) \mid A\rangle\langle A \mid \psi(t)\rangle .
$$

Therefore, the rate of change in time of the modulus squared of the quantum overlap $\langle\psi(t) \mid A\rangle$ becomes:

$$
\begin{aligned}
\frac{d\left[|\langle\psi(t) \mid A\rangle|^{2}\right]}{d t} & =\langle\dot{\psi} \mid A\rangle\langle A \mid \psi\rangle+\langle\psi \mid A\rangle\langle A \mid \dot{\psi}\rangle \\
& =\langle\psi \mid A\rangle\langle A \mid \dot{\psi}\rangle+\langle\psi \mid A\rangle^{*}\langle A \mid \dot{\psi}\rangle^{*} \\
& =2 \operatorname{Re}[\langle\psi \mid A\rangle\langle A \mid \dot{\psi}\rangle],
\end{aligned}
$$

where $|\dot{\psi}\rangle=\left|\frac{d \psi}{d t}\right\rangle=\frac{d|\psi\rangle}{d t}$. Using Equation (8) with $\hat{Q}=\hat{\mathrm{H}}$ together with Equation (10), we obtain:

$$
|\dot{\psi}\rangle=-\frac{i}{\hbar} \hat{\mathrm{H}}|\psi\rangle=-\frac{i}{\hbar}\left[\langle\hat{\mathrm{H}}\rangle|\psi\rangle+\Delta E\left|\psi_{\perp}\right\rangle\right]
$$


Using Equation (13), Equation (12) becomes:

$$
\begin{aligned}
\frac{d\left[|\langle\psi(t) \mid A\rangle|^{2}\right]}{d t} & =2 \operatorname{Re}\left\{\langle\psi \mid A\rangle \cdot\left(-\frac{i}{\hbar}\right) \cdot\left[\langle\hat{\mathrm{H}}\rangle\langle A \mid \psi\rangle+\Delta E\left\langle A \mid \psi_{\perp}\right\rangle\right]\right\} \\
& =2 \operatorname{Re}\left[-\frac{i}{\hbar}\langle\hat{\mathrm{H}}\rangle|\langle\psi \mid A\rangle|^{2}-\frac{i}{\hbar} \Delta E\langle\psi \mid A\rangle\left\langle A \mid \psi_{\perp}\right\rangle\right] \\
& =-2 \frac{\Delta E}{\hbar} \operatorname{Re}\left[i\langle\psi \mid A\rangle\left\langle A \mid \psi_{\perp}\right\rangle\right],
\end{aligned}
$$

that is:

$$
\frac{d\left[|\langle\psi(t) \mid A\rangle|^{2}\right]}{d t}=-2 \frac{\Delta E}{\hbar} \operatorname{Re}\left[i\langle\psi \mid A\rangle\left\langle A \mid \psi_{\perp}\right\rangle\right] .
$$

For a given value of $\Delta E$ and $|\langle\psi \mid A\rangle|$, the absolute value of the RHS of Equation (15) achieves its maximum number when $\left|\left\langle A \mid \psi_{\perp}\right\rangle\right|$ is maximum by observing that:

$$
\left|\operatorname{Re}\left[i\langle\psi \mid A\rangle\left\langle A \mid \psi_{\perp}\right\rangle\right]\right| \leq\left|i\langle\psi \mid A\rangle\left\langle A \mid \psi_{\perp}\right\rangle\right| \leq|\langle\psi \mid A\rangle|\left|\left\langle A \mid \psi_{\perp}\right\rangle\right| .
$$

To determine the maximum of $\left|\left\langle A \mid \psi_{\perp}\right\rangle\right|$, we proceed as follows. In general, the resolution of the identity for a $n$-dimensional Hilbert space $\mathcal{H}$ is given by

$$
\hat{1}=\sum_{i=1}^{n}\left|\psi_{i}\right\rangle\left\langle\psi_{i}\right|
$$

with $\left\langle\psi_{i} \mid \psi_{j}\right\rangle=\delta_{i j}$ for any $1 \leq i, j \leq n$. For clarity of exposition and without loss of generality, we assume here a resolution of the identity on the full Hilbert space $\mathcal{H}$ that can be recast as $\hat{1} \stackrel{\text { def }}{=}|\psi\rangle\left\langle\psi|+| \psi_{\perp}\right\rangle\left\langle\psi_{\perp}|+| \psi_{\perp \perp}\right\rangle\left\langle\psi_{\perp \perp}\right|$. We note that:

$$
\begin{aligned}
|A\rangle & =\hat{1}|A\rangle \\
& =\left[|\psi\rangle\left\langle\psi|+| \psi_{\perp}\right\rangle\left\langle\psi_{\perp}|+| \psi_{\perp \perp}\right\rangle\left\langle\psi_{\perp \perp}\right|\right]|A\rangle \\
& =\langle\psi \mid A\rangle|\psi\rangle+\left\langle\psi_{\perp} \mid A\right\rangle\left|\psi_{\perp}\right\rangle+\left\langle\psi_{\perp \perp} \mid A\right\rangle\left|\psi_{\perp \perp}\right\rangle,
\end{aligned}
$$

where:

$$
\left\langle\psi(t) \mid \psi_{\perp}(t)\right\rangle=\left\langle\psi(t) \mid \psi_{\perp \perp}(t)\right\rangle=\left\langle\psi_{\perp}(t) \mid \psi_{\perp \perp}(t)\right\rangle=0, \forall t .
$$

Since $\langle A \mid A\rangle=1$, from Equations (18) and (19), we obtain:

$$
|\langle\psi \mid A\rangle|^{2}+\left|\left\langle\psi_{\perp} \mid A\right\rangle\right|^{2}+\left|\left\langle\psi_{\perp \perp} \mid A\right\rangle\right|^{2}=1
$$

Therefore, we obtain from Equation (20) that:

$$
\left|\left\langle A \mid \psi_{\perp}\right\rangle\right|^{2}=1-|\langle\psi \mid A\rangle|^{2}-\left|\left\langle\psi_{\perp \perp} \mid A\right\rangle\right|^{2},
$$

that is, $\left|\left\langle A \mid \psi_{\perp}\right\rangle\right|$ is maximum when $\left\langle\psi_{\perp \perp} \mid A\right\rangle=0$. Its maximum value equals:

$$
\left|\left\langle A \mid \psi_{\perp}\right\rangle\right|_{\max }=\sqrt{1-|\langle A \mid \psi\rangle|^{2}} .
$$

For completeness, we note that, in the general scenario where one employs Equation (17), $\left|\left\langle A \mid \psi_{\perp}\right\rangle\right|$ is maximum when $\left\langle\psi_{i} \mid A\right\rangle=0$ for any $i=3, \ldots, n$ with $\left|\psi_{1}\right\rangle$ and $\left|\psi_{2}\right\rangle$ corresponding to $|\psi\rangle$ and $\left|\psi_{\perp}\right\rangle$, respectively.

Before continuing our proof, we would like to remark at this point that it is straightforward to see that the subscript "max" in Equation (22) is not strictly necessary if we employ a different resolution of the identity operator $\hat{1}$ on the full Hilbert space with the orthogonal decomposition given by $\mathcal{H} \stackrel{\text { def }}{=} \mathcal{H}_{\psi} \oplus \mathcal{H}_{\psi_{\perp}}$ as used in Appendix $\mathrm{A}$, and not by $\mathcal{H} \stackrel{\text { def }}{=} \mathcal{H}_{\psi} \oplus \mathcal{H}_{\psi_{\perp}} \oplus \mathcal{H}_{\psi_{\perp \perp}}$ as in Equation (18). 
Returning to our proof, we note from Equations (15) and (22) that the maximum of the absolute value of the rate of change of the quantum overlap only depends on $\Delta E$ and $|\langle A \mid \psi\rangle|$ and is given by

$$
\left|\frac{d\left[|\langle\psi(t) \mid A\rangle|^{2}\right]}{d t}\right|_{\max }=2 \frac{\Delta E}{\hbar}|\langle A \mid \psi\rangle| \sqrt{1-|\langle A \mid \psi\rangle|^{2}} .
$$

Finally, to obtain the fastest evolution to a nonorthogonal state, we impose:

$$
\frac{d\left[|\langle\psi \mid A\rangle|^{2}\right]}{d t}=-2 \frac{\Delta E}{\hbar}|\langle A \mid \psi\rangle| \sqrt{1-|\langle A \mid \psi\rangle|^{2}} .
$$

For the sake of completeness, we point out that this constraint entails imposing a phase relationship between $\langle\psi \mid A\rangle=|\langle\psi \mid A\rangle| e^{i \varphi_{\psi}}$ and $\left\langle\psi_{\perp} \mid A\right\rangle=\left|\left\langle\psi_{\perp} \mid A\right\rangle\right| e^{i \varphi_{\psi_{\perp}}}$ such that $\varphi_{\psi_{\perp}}-\varphi_{\psi}=\pi / 2$. Letting $|\langle\psi \mid A\rangle| \stackrel{\text { def }}{=} \cos (\theta)$, Equation (24) yields:

$$
\frac{d\left[\cos ^{2}(\theta)\right]}{d t}=-2 \frac{\Delta E}{\hbar} \cos (\theta) \sin (\theta)
$$

that is:

$$
-2 \cos (\theta) \sin (\theta) \dot{\theta}=-2 \frac{\Delta E}{\hbar} \cos (\theta) \sin (\theta) .
$$

Thus, we obtain from Equation (26) that:

$$
\dot{\theta}=\frac{\Delta E}{\hbar} .
$$

Integrating Equation (27), we obtain:

$$
\int_{\theta(0)}^{\theta\left(T_{A B}\right)} d \theta=\int_{0}^{T_{A B}} \frac{\Delta E}{\hbar} d t
$$

Recalling that $|\langle\psi(t) \mid A\rangle| \stackrel{\text { def }}{=} \cos [\theta(t)]$ with $\theta(0)=0$ since $|\psi(0)\rangle \stackrel{\text { def }}{=}|A\rangle$, after some simple algebra, we finally obtain from Equation (28):

$$
T_{A B}=\frac{\hbar}{\Delta E} \cos ^{-1}[|\langle A \mid B\rangle|]
$$

that is:

$$
\Delta E \cdot T_{A B}=\hbar \cos ^{-1}[|\langle A \mid B\rangle|] .
$$

The quantity $T_{A B}$ in Equation (29) denotes the minimum time interval needed for the evolution (unitary Schrödinger evolution with the assumption of the constant dispersion of the Hamiltonian operator) from $|A\rangle$ to $|B\rangle$ with the two states being nonorthogonal. As a side remark, we point out that when $|A\rangle$ and $|B\rangle$ are orthogonal, Equation (29) yields:

$$
\Delta E \cdot T_{A B}^{\perp}=\frac{h}{4} .
$$

Equation (31) is the result that was originally obtained without use of geometrical reasoning by Vaidman in Ref. [4]. As a final remark, we point out that Equation (29) can be recast as

$$
T_{A B}=\frac{\hbar}{\Delta E} \sin ^{-1}\left[\sqrt{1-|\langle A \mid B\rangle|^{2}}\right] .
$$

Equation (32) reduces to the optimal time expression obtained by Bender and collaborators in Ref. [13] when setting $|A\rangle \stackrel{\text { def }}{=}|0\rangle$ and $|B\rangle \stackrel{\text { def }}{=} a|0\rangle+b|1\rangle$ with $a, b \in \mathbb{C}$. 
As pointed out below Equation (9), our demonstration leading to Equation (28) works equally well for time-dependent Hamiltonians $\hat{\mathrm{H}}(t)$. In particular, Equation (28) remains valid if we replace a constant $\Delta E$ with a time-dependent $\Delta E(t)$. In this case, the integration of Equation (28) yields:

$$
\langle\Delta E\rangle_{T_{A B}} T_{A B}=\hbar \cos ^{-1}[|\langle A \mid B\rangle|],
$$

where $\langle\Delta E\rangle_{T_{A B}}$ is the time-averaged uncertainty during the time interval $T_{A B}$ defined as

$$
\langle\Delta E\rangle_{T_{A B}} \stackrel{\text { def }}{=} \frac{1}{T_{A B}} \int_{0}^{T_{A B}} \Delta E\left(t^{\prime}\right) d t^{\prime}=\frac{\mathcal{E}\left(T_{A B}\right)-\mathcal{E}(0)}{T_{A B}},
$$

with $d \mathcal{E} / d t^{\prime} \stackrel{\text { def }}{=} \Delta E\left(t^{\prime}\right)$. It is clear from Equations (33) and (34) that $\mathcal{E}\left(T_{A B}\right)=\mathcal{E}(0)+$ $\hbar \cos ^{-1}[|\langle A \mid B\rangle|]$ and, therefore, we expect that a closed form analytical expression of the minimum evolution time $T_{A B}$ cannot be generally obtained in an explicit manner in such more realistic time-dependent scenarios.

\section{Efficiency with Geometric Arguments}

Recall that in the geometric formulation of quantum mechanical Schrödinger's evolution, one can consider a measure of efficiency that quantifies the departure of an effective (non-geodesic evolution, in general,) from an ideal geodesic evolution. Such a geodesic evolution is characterized by paths of shortest length that connect initial and final quantum states $|A\rangle$ and $|B\rangle$, respectively. In particular, under this scheme, one can define an efficiency in geometric quantum mechanics that takes into account a quantum mechanical evolution of a state vector $|\psi(t)\rangle$ in an $N$-dimensional complex Hilbert space specified by the Schrödinger equation:

$$
i \hbar \partial_{t}|\psi(t)\rangle=\hat{\mathrm{H}}(t)|\psi(t)\rangle,
$$

with $0 \leq t \leq T_{A B}, \hbar \stackrel{\text { def }}{=} h /(2 \pi), h$ being the Planck constant, and $\hat{H}$ denoting the Hamiltonian of the system. A geometric measure of efficiency $\eta_{\mathrm{QM}}^{\text {(geometric) }}$ with $0 \leq \eta_{\mathrm{QM}}^{\text {(geometric) }} \leq 1$ for such a quantum system can be defined as $[3,14]$

$$
\eta_{\mathrm{QM}}^{\text {(geometric) }} \stackrel{\text { def }}{=} \frac{s_{0}}{s}=1-\frac{\Delta s}{s}=\frac{2 \cos ^{-1}\left[\left|\left\langle\psi(0) \mid \psi\left(T_{A B}\right)\right\rangle\right|\right]}{2 \int_{0}^{T_{A B}} \frac{\Delta E\left(t^{\prime}\right)}{\hbar} d t^{\prime}},
$$

where $\Delta s \stackrel{\text { def }}{=} s-s_{0}, s_{0} \stackrel{\text { def }}{=} 2 \cos ^{-1}\left[\left|\left\langle\psi(0) \mid \psi\left(T_{A B}\right)\right\rangle\right|\right]$ denotes the distance along the (ideal) shortest geodesic path joining the distinct initial $|\psi(0)\rangle \stackrel{\text { def }}{=}|A\rangle$ and final $\left|\psi\left(T_{A B}\right)\right\rangle \stackrel{\text { def }}{=}|B\rangle$ states on the projective Hilbert space $\mathbb{C} P^{N-1}$ and finally, $s \stackrel{\text { def }}{=} 2 \int_{0}^{T_{A B}}\left[\Delta E\left(t^{\prime}\right)\right] / \hbar d t^{\prime}$ is the distance along the (real) actual dynamical trajectory traced by the state vector $|\psi(t)\rangle$ with $0 \leq t \leq T_{A B}$ and finally, $\Delta E$ represents the uncertainty in the energy of the system. We emphasize that the numerator in Equation (36) is the angle between the state vectors $|\psi(0)\rangle$ and $\left|\psi\left(T_{A B}\right)\right\rangle$ and is equal to the Wootters distance $d s_{\text {Wootters }}[15]$ :

$$
d s_{\text {Wootters }}\left(|\psi(0)\rangle,\left|\psi\left(T_{A B}\right)\right\rangle\right) \stackrel{\text { def }}{=} 2 \cos ^{-1}\left[\left|\left\langle\psi(0) \mid \psi\left(T_{A B}\right)\right\rangle\right|\right] .
$$

Furthermore, the denominator in Equation (36) represents the integral of the infinitesimal distance $d s$ along the evolution curve in the projective Hilbert space [3]:

$$
d s \stackrel{\text { def }}{=} 2 \frac{\Delta E(t)}{\hbar} d t
$$

Curiously, Anandan and Aharonov proved that the infinitesimal distance $d s$ in is connected to the Fubini-Study infinitesimal distance $d s_{\text {Fubini-Study }}$ by the following condition:

$$
d s_{\text {Fubini-Study }}^{2}(|\psi(t)\rangle,|\psi(t+d t)\rangle) \stackrel{\text { def }}{=} 4\left[1-|\langle\psi(t) \mid \psi(t+d t)\rangle|^{2}\right]=4 \frac{\Delta E^{2}(t)}{\hbar^{2}} d t^{2}+\mathcal{O}\left(d t^{3}\right),
$$


with $\mathcal{O}\left(d t^{3}\right)$ denoting an infinitesimal quantity equal or higher than $d t^{3}$. Equations (38) and (39) imply that $s$ is proportional to the time integral of the uncertainty in energy $\Delta E$ of the system and specifies the distance along the quantum evolution of the system in the projective Hilbert space as measured by the Fubini-Study metric. We point out that when the actual dynamical curve coincides with the shortest geodesic path connecting the initial and final states, $\Delta s$ is equal to zero and the efficiency $\eta_{\mathrm{QM}}^{\text {(geometric) }}$ in Equation (36) equals one. Obviously, $\pi$ is the shortest possible distance between two orthogonal states in the projective Hilbert space. In general, however, $s \geq \pi$ for such a pair of orthogonal pure states.

Given the important role played by energy uncertainty in the geometry of quantum evolutions, one may wonder whether or not there is some sort of quantum mechanical uncertainty relation in this geometric framework. We recall that the standard quantum mechanical uncertainty relation is given by [16]

$$
\Delta x \Delta p \geq \hbar / 2
$$

Equation (40) mirrors the intrinsic randomness of the outcomes of quantum experiments. Precisely, if one repeats several times the same state preparation scheme and then measures the operators $x$ or $p$, the observations collected for $x$ and $p$ are specified by standard deviations $\Delta x$ and $\Delta p$ whose product $\Delta x \Delta p$ is greater than $\hbar / 2$. Gaussian wave packets, in particular, are characterized by a minimum position-momentum uncertainty defined by $\Delta x \Delta p=\hbar / 2$. In the geometry of quantum evolutions, there is an analogue of Equation (40) where, for instance, Gaussian wave packets are replaced by geodesic paths in the projective Hilbert space. Indeed, taking into consideration the time-averaged uncertainty in energy $\langle\Delta E\rangle_{T_{A B}^{\perp}}$ during a time interval $T_{A B}^{\perp}$ defined as [3]

$$
\langle\Delta E\rangle_{T_{A B}^{\perp}} \stackrel{\text { def }}{=} \frac{1}{T_{A B}^{\perp}} \int_{0}^{T_{A B}^{\perp}} \Delta E\left(t^{\prime}\right) d t^{\prime} .
$$

The quantity $T_{A B}^{\perp}$ in Equation (41) defines the orthogonalization time, that is, the time interval during which the system passes from an initial state $|A\rangle \stackrel{\text { def }}{=}|\psi(0)\rangle$ to a final state $|B\rangle \stackrel{\text { def }}{=}\left|\psi\left(T_{A B}^{\perp}\right)\right\rangle$ where $\langle B \mid A\rangle=\delta_{A B}$. Employing Equations (38) and (41) and remembering that the shortest possible distance between two orthogonal quantum states in the projective Hilbert space is $\pi$, we obtain:

$$
\langle\Delta E\rangle_{T_{A B}^{\perp}} T_{A B}^{\perp} \geq h / 4 .
$$

Specifically, the equality in Equation (42) only holds when the quantum evolution is a geodesic evolution. Therefore, geodesic paths represent minimum time-averaged energy uncertainty trajectories just as Gaussian wave packets specify minimum positionmomentum uncertainty wave packets. Summarizing, when a quantum evolution exhibits minimum uncertainty $\langle\Delta E\rangle_{T_{A B}^{\perp}} T_{A B}^{\perp}=h / 4$, unit efficiency $\eta_{\mathrm{QM}}^{\text {(geometric) }}=1$ is achieved. This, in turn, only occurs if the physical systems move along a geodesic path in the projective Hilbert space. Interestingly, the Anandan-Aharonov time-energy uncertainty relation in Equation (42) is related to the statistical speed of the evolution $d s_{\mathrm{FS}} / d t$ of the physical system with $d s_{\mathrm{FS}}^{2}$ being the Fubini-Study infinitesimal line element squared. Precisely, since $d s_{\mathrm{FS}} / d t$ is proportional to $\Delta E$, the system moves rapidly wherever the uncertainty in energy assumes large values [17].

We observe that if $|A\rangle$ and $|B\rangle$ are orthogonal, and in addition, $\Delta E$ is constant, we have from Equation (36) that:

$$
\eta_{\mathrm{QM}}^{\text {(geometric) }}=\frac{2 \frac{\pi}{2}}{2 \frac{\Delta E}{\hbar} T_{A B}^{\perp}}=\frac{h}{4} \frac{1}{\Delta E \cdot T_{A B}^{\perp}} .
$$


Therefore, from Equation (43), we obtain:

$$
\eta_{\mathrm{QM}}^{\text {(geometric) }} \leq 1 \Leftrightarrow \frac{h}{4} \frac{1}{\Delta E \cdot T_{A B}^{\perp}} \leq 1 \Leftrightarrow \Delta E \cdot T_{A B}^{\perp} \geq \frac{h}{4}
$$

In particular, we obtain:

$$
\text { geodesic motion } \Leftrightarrow \eta_{\mathrm{QM}}^{\text {(geometric) }}=1 \Leftrightarrow \frac{h}{4} \frac{1}{\Delta E \cdot T_{A B}^{\perp}}=1 \Leftrightarrow \Delta E \cdot T_{A B}^{\perp}=\frac{h}{4},
$$

that is:

$$
\text { geodesic motion } \Leftrightarrow \Delta E \cdot T_{A B}^{\perp}=\frac{h}{4} \text {. }
$$

We remark that the geodesic constraint in Equation (46) is not a time-energy uncertainty condition. Instead, it simply states that distance $=$ speed $\times$ time. Indeed, using the Anandan-Aharonov relation that states that the (angular) speed $v$ of a unitary evolution is proportional to the energy uncertainty $\Delta E[3,18], v=(2 \Delta E) / \hbar$, the condition $\Delta E \cdot T_{A B}^{\perp}=h / 4$ can be recast as $s_{0}=v T_{A B}^{\perp}$ with $s_{0} \stackrel{\text { def }}{=} \pi$. Furthermore, if $|A\rangle$ and $|B\rangle$ are nonorthogonal and in addition, $\Delta E$ is constant, we have from Equation (36) that:

$$
\eta_{\mathrm{QM}}^{\text {(geometric) }}=\frac{\hbar}{\Delta E \cdot T_{A B}} \cos ^{-1}[|\langle A \mid B\rangle|] .
$$

Therefore, Equation (47) yields:

$$
\eta_{\mathrm{QM}}^{\text {(geometric) }} \leq 1 \Leftrightarrow T_{A B} \geq \frac{\hbar}{\Delta E} \cos ^{-1}[|\langle A \mid B\rangle|]
$$

that is:

$$
\Delta E \cdot T_{A B} \geq \hbar \cos ^{-1}[|\langle A \mid B\rangle|] .
$$

Equation (49) generalizes the inequality $\Delta E \cdot T_{A B}^{\perp} \geq h / 4$ in Equation (44) and is generally valid even when the quantum system does not pass through orthogonal states. Moreover, the inequality in Equation (49) is in agreement with Equation (29) derived in the previous Section without any geometrical consideration. The derivation of Equation (49) provides a simple quantitative justification of the verbal statement made by Anandan and Aharonov in Ref. [3] concerning the validity of the inequality $\varepsilon \leq 1$ extended to a system that does not pass through orthogonal states. In particular, we have from Equation (49) that:

geodesic motion $\Leftrightarrow \eta_{\mathrm{QM}}^{\text {(geometric) }}=1 \Leftrightarrow \frac{\hbar}{\Delta E \cdot T_{A B}} \cos ^{-1}[|\langle A \mid B\rangle|]=1 \Leftrightarrow \Delta E \cdot T_{A B}=\hbar \cos ^{-1}[|\langle A \mid B\rangle|]$,

that is:

$$
\text { geodesic motion } \Leftrightarrow \Delta E \cdot T_{A B}=\hbar \cos ^{-1}[|\langle A \mid B\rangle|] \text {. }
$$

We emphasize that the inequality $\eta_{\mathrm{QM}}^{\text {(geometric) }} \leq 1$ in Equation (48) also holds true in the time-dependent Hamiltonian scenario by simply applying our line of reasoning developed for the time-independent scenario and replacing $\Delta E$ with $\langle\Delta E\rangle_{T_{A B}}$ as defined in Equation (34). Specifically, the time-dependent version of Equation (51) for non-geodesic motion is specified by the inequality $\langle\Delta E\rangle_{T_{A B}} T_{A B} \geq \hbar \cos ^{-1}[|\langle A \mid B\rangle|]$.

Interestingly, we also point out that the energy dispersion $\Delta E$ (as defined in Equation (9)) of a constant Hamiltonian operator $\hat{H}$ describing a two-level quantum system with spectral decomposition given by $\hat{H} \stackrel{\text { def }}{=} E_{1}\left|E_{1}\right\rangle\left\langle E_{1}\left|+E_{2}\right| E_{2}\right\rangle\left\langle E_{2}\right|$ (where $E_{2} \geq E_{1}$ and $\left\langle E_{i} \mid E_{j}\right\rangle=\delta_{i j}$ ) with respect to the normalized initial state $|A\rangle$ is given by

$$
\Delta E=\frac{E_{2}-E_{1}}{2} \sqrt{1-\left(\left|\alpha_{1}\right|^{2}-\left|\alpha_{2}\right|^{2}\right)^{2}},
$$


once $|A\rangle$ is decomposed as $\alpha_{1}\left|E_{1}\right\rangle+\alpha_{2}\left|E_{2}\right\rangle$ with $\alpha_{1}, \alpha_{2} \in \mathbb{C}$. From Equation (52), we note that the maximum value of $\Delta E$ is obtained for $\left|\alpha_{1}\right|=\left|\alpha_{2}\right|$ where $\left|\alpha_{1}\right| \stackrel{\text { def }}{=}\left|\left\langle E_{1} \mid A\right\rangle\right|$ and $\left|\alpha_{2}\right| \stackrel{\text { def }}{=}\left|\left\langle E_{2} \mid A\right\rangle\right|$, respectively. Moreover, this maximum value equals $\Delta E_{\max } \stackrel{\text { def }}{=}$ $\left(E_{2}-E_{1}\right) / 2$. Therefore, the minimum evolution time $T_{A B}$ from an initial state $|A\rangle$ to a final state $|B\rangle$ becomes:

$$
T_{A B}^{\min }=\frac{2 \hbar}{E_{2}-E_{1}} \cos ^{-1}[|\langle A \mid B\rangle|] .
$$

Finally, when the quantum evolution is between the orthogonal initial and final states $|A\rangle$ and $|B\rangle$, Equation (53) yields $T_{A B}^{\perp, \min }=h /\left[2\left(E_{2}-E_{1}\right)\right]$. Clearly, from Equations (51) and (53), we observe that the travel time $T_{A B}$ depends on the Hamiltonian $\mathrm{H}$ through the energy uncertainty $\Delta E$. Specifically, $T_{A B}$ can be made arbitrarily small if $\Delta E$ can be made arbitrarily large. However, in typical physical scenarios specified by a finite-dimensional Hilbert space with temporally bounded energy eigenvalues $\left\{E_{n}(t)\right\}$ [19], the dispersion of the Hamiltonian operator is upper bounded. Specifically, it happens that $\Delta E \leq \mathcal{E}_{\max }$ if for any $n \in \mathbb{N}$ and for any $t$, one imposes $\left|E_{n}(t)\right| \leq \mathcal{E}_{\max }$ for some $\mathcal{E}_{\max } \in \mathbb{R}_{+}$. Thus, the minimum time travel is lower bounded with $T_{A B}^{\min } \geq\left(\hbar / \mathcal{E}_{\max }\right) \cos ^{-1}[|\langle A \mid B\rangle|]$. In Table 1 , we summarize our results for optimal quantum evolution conditions between both orthogonal and nonorthogonal states. In the next section, we present two explicit examples.

Table 1. Schematic description of the optimal quantum evolution conditions for motion between either two orthogonal or nonorthogonal states. In both scenarios, the energy dispersion $\Delta E^{2}$ of the Hamiltonian operator $\mathrm{H}$ is assumed to be constant in time. Unit geometric quantum efficiency motion with $\eta \stackrel{\text { def }}{=} \eta_{\mathrm{QM}}^{\text {(geometric) }}=1$ is only achieved when the time-energy inequality relation becomes an equality constraint.

\begin{tabular}{ccc}
\hline Quantum States & Time-Energy Inequality Constraint & Optimal Quantum Evolution Condition \\
\hline Orthogonal & $T_{A B}^{\perp} \geq h /(4 \Delta E)$, with $0 \leq \eta \leq 1$ & $T_{A B}^{\perp, \min }=h /\left(4 \Delta E_{\max }\right)$, with $\eta=1$ \\
Nonorthogonal & $T_{A B} \geq \hbar \cos ^{-1}[|\langle A \mid B\rangle|] / \Delta E$, with $0 \leq \eta \leq 1$ & $T_{A B}^{\text {min }}=\hbar \cos ^{-1}[|\langle A \mid B\rangle|] / \Delta E_{\max }$, with $\eta=1$ \\
\hline
\end{tabular}

\section{Applications}

In this section, we explicitly discuss the notions of minimum evolution time and quantum geometric efficiency in two examples.

\subsection{Time-Independent Scenario}

In the first scenario, we consider a physical system characterized by a time-independent Hamiltonian:

$$
\mathrm{H} \stackrel{\text { def }}{=} \epsilon \sigma_{x}=\left(\begin{array}{ll}
0 & \epsilon \\
\epsilon & 0
\end{array}\right),
$$

with $\epsilon>0$ denoting the strength of the Hamiltonian and $\sigma_{x}$ being the usual Pauli matrix. The unitary evolution operator $U(t) \stackrel{\text { def }}{=} e^{-\frac{i}{\hbar} \mathrm{H} t}$ corresponding to the Hamiltonian in Equation (54) is given by

$$
U(t)=\cos \left(\frac{\epsilon}{\hbar} t\right) I-i \sin \left(\frac{\epsilon}{\hbar} t\right) \sigma_{x}=\left(\begin{array}{cc}
\cos \left(\frac{\epsilon}{\hbar} t\right) & -i \sin \left(\frac{\epsilon}{\hbar} t\right) \\
-i \sin \left(\frac{e}{\hbar} t\right) & \cos \left(\frac{\epsilon}{\hbar} t\right)
\end{array}\right),
$$

with $I$ being the $2 \times 2$ identity matrix. Let us consider the quantum mechanical evolution from $|A\rangle \stackrel{\text { def }}{=}|0\rangle$ to $|B\rangle \stackrel{\text { def }}{=}-i|1\rangle$ along the path $\gamma_{t}: t \mapsto|\psi(t)\rangle$ with $|\psi(t)\rangle \stackrel{\text { def }}{=} U(t)|A\rangle$ and $0 \leq t \leq T_{A B}^{\text {(effective) }}$ with $T_{A B}^{(\text {(effective) }} \stackrel{\text { def }}{=} \frac{\pi}{2 \epsilon} \hbar$. Note that $|A\rangle \stackrel{\text { def }}{=}|\psi(0)\rangle=|0\rangle$ and $|B\rangle \stackrel{\text { def }}{=}\left|\psi\left(\frac{\pi}{2 \epsilon} \hbar\right)\right\rangle=-i|1\rangle$ is physically equivalent to $|1\rangle$.

To begin, we observe that the path $\gamma_{t}$ traced by the state vector $|\psi(t)\rangle=\cos \left(\frac{\epsilon}{\hbar} t\right)|A\rangle+$ $\sin \left(\frac{\epsilon}{\hbar} t\right)|B\rangle$ is a geodesic path. Indeed, a simple calculation shows that $|\psi(t)\rangle$ can be recast as a quantum geodesic line [20]: 


$$
|\psi(t)\rangle=|\tilde{\psi}(\xi(t))\rangle \stackrel{\text { def }}{=} \mathcal{N}_{\xi}[(1-\xi)|A\rangle+\xi|B\rangle],
$$

where $\mathcal{N}_{\xi} \stackrel{\text { def }}{=}[1-2 \xi(1-\xi)]^{-1 / 2}$ is a normalization constant, while $\xi(t)$ denotes a strictly monotonic function of $t$ given by

$$
\xi(t) \stackrel{\text { def }}{=} \frac{\tan \left(\frac{\epsilon}{\hbar} t\right)}{1+\tan \left(\frac{\epsilon}{\hbar} t\right)},
$$

with $0 \leq \xi(t) \leq 1$. The geodesic nature of the path $\gamma_{t}$ can also be explained in terms of the energy spread and the efficiency concepts. Indeed, we have $\Delta E^{2}=\epsilon^{2}, T_{A B}^{(\text {effective) }}=\frac{\pi}{2 \epsilon} \hbar$, and $\langle A \mid B\rangle=0$. Therefore, the minimum evolution constraint condition $\Delta E \cdot T_{A B}^{(\text {ideal })}=h / 4$ yields:

$$
T_{A B}^{(\text {effective })}=T_{A B}^{(\text {ideal })} .
$$

Finally, the quantum motion occurs with unit geometric efficiency $\eta_{\mathrm{QM}}^{\text {(geometric) }}=1$.

\subsection{Time-Dependent Scenario}

In the second scenario, we take into consideration a physical system specified by means of a time-dependent Hamiltonian:

$$
\mathrm{H}(t) \stackrel{\text { def }}{=} \epsilon \cos (\omega t) \sigma_{x}+\epsilon \sin (\omega t) \sigma_{y}+\frac{\hbar \omega_{0}}{2} \sigma_{z}=\left(\begin{array}{cc}
\frac{\hbar \omega_{0}}{2} & \epsilon e^{-i \omega t} \\
\epsilon e^{i \omega t} & -\frac{\hbar \omega_{0}}{2}
\end{array}\right),
$$

with $\epsilon>0, \omega>0$, and $\hbar \omega_{0}>0$ denoting the strength of the external drive, the angular frequency of the external drive, and the energy difference between the two states of the two-state quantum system (that is: $\hbar \omega_{0} \stackrel{\text { def }}{=} E_{2}-E_{1}>0$ ). We define the detuning of the driving field from resonance as $\Delta \stackrel{\text { def }}{=} \hbar\left(\omega-\omega_{0}\right)$. Clearly, $\sigma_{x}, \sigma_{y}$, and $\sigma_{z}$ are the usual Pauli matrices. The Hamiltonian in Equation (59) emerges in the context of the nearresonance phenomenon in a two-state quantum system. The unitary evolution operator $U(t)$ corresponding to $\mathrm{H}(t)$ in Equation (59) is [21]:

$$
\begin{aligned}
U(t) & =\cos \left(\frac{\kappa}{\hbar} t\right) I-i \sin \left(\frac{\kappa}{\hbar} t\right)\left[\frac{\Delta}{2 \kappa} \sigma_{z}+\frac{\epsilon}{\kappa} \sigma_{x}\right] \\
& =\left(\begin{array}{cc}
\cos \left(\frac{\kappa}{\hbar} t\right)-i \frac{\Delta}{2 \kappa} \sin \left(\frac{\kappa}{\hbar} t\right) & -i \frac{\epsilon}{\kappa} \sin \left(\frac{\kappa}{\hbar} t\right) \\
-i \frac{\epsilon}{\kappa} \sin \left(\frac{\kappa}{\hbar} t\right) & \cos \left(\frac{\kappa}{\hbar} t\right)+i \frac{\Delta}{2 \kappa} \sin \left(\frac{\kappa}{\hbar} t\right)
\end{array}\right),
\end{aligned}
$$

where $\kappa \stackrel{\text { def }}{=} \sqrt{\epsilon^{2}+\Delta^{2} / 4}$. Let us consider the quantum mechanical evolution from $|A\rangle \stackrel{\text { def }}{=}|0\rangle$

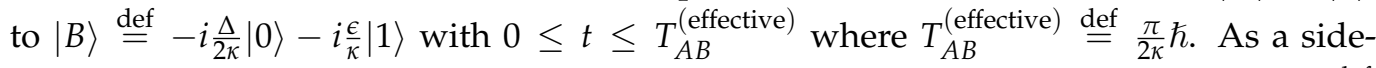
note, we note that near-resonance, $|\Delta| \ll \epsilon$ and $\kappa \rightarrow \epsilon$. Therefore, $\frac{\pi}{2 \kappa} \hbar \rightarrow \frac{\pi}{2 \epsilon} \hbar \stackrel{\text { def }}{=}$ $\left[T_{A B}^{\text {(effective) })}\right]_{\text {first-scenario }}$, and $-i \frac{\Delta}{2 \kappa}|0\rangle-i \frac{\epsilon}{\kappa}|1\rangle \rightarrow-i|1\rangle \stackrel{\text { def }}{=}[|B\rangle]_{\text {first-scenario }}$.

To begin, we note that the path $\gamma_{t}$ traced by the state vector $|\psi(t)\rangle=\cos \left(\frac{\kappa}{\hbar} t\right)|A\rangle+$ $\sin \left(\frac{\kappa}{\hbar} t\right)|B\rangle$ with $\langle A \mid B\rangle=-i \frac{\Delta}{2 \kappa} \neq 0$ is not a geodesic path. Indeed, following the reasoning outlined in the first example, a simple calculation shows that $|\psi(t)\rangle$ cannot be recast as a quantum geodesic line. The non-geodesic nature of the path $\gamma_{t}$ can also be understood by studying the expression of the energy spread of the system. Indeed, from a simple calculation, we obtain:

$$
\Delta E^{2}(t)=\varepsilon^{2}+\frac{\left(\hbar \omega_{0}\right)^{2}}{4}-\left\{\begin{array}{c}
\frac{\hbar \omega_{0}}{2}\left[\cos ^{2}\left(\frac{\kappa}{\hbar} t\right)-\frac{4 \varepsilon^{2}-\Delta^{2}}{4 \kappa^{2}} \sin ^{2}\left(\frac{\kappa}{\hbar} t\right)\right]-\frac{\varepsilon^{2}}{\kappa} \sin \left(2 \frac{\kappa}{\hbar} t\right) \sin (\omega t)+ \\
+\frac{2 \varepsilon^{2}}{\kappa} \frac{\Delta}{2 \kappa} \sin ^{2}\left(\frac{\kappa}{\hbar} t\right) \cos (\omega t)
\end{array}\right\}^{2} .
$$


At this point, to show the non-geodesic behavior of the evolution path $\gamma_{t}$ in this second scenario, we focus on the short-time limit of $\Delta E^{2}(t)$ in the near-resonance case. First, assuming $|\Delta| \ll \epsilon, \Delta E^{2}(t)$ in Equation (61) reduces to:

$$
\Delta E^{2}(t)=\varepsilon^{2}+\frac{\left(\hbar \omega_{0}\right)^{2}}{4}\left\{1-\left[\cos \left(2 \frac{\varepsilon}{\hbar} t\right)-\frac{2 \varepsilon}{\hbar \omega_{0}} \sin \left(2 \frac{\varepsilon}{\hbar} t\right) \sin (\omega t)\right]^{2}\right\},
$$

with $0 \leq t \leq \frac{\pi}{2 \epsilon} \hbar$. Before considering the short-time limit, we make a few considerations that motivate the consideration of this limit. The strength of the external drive $\epsilon$ is related to the Rabi angular frequency by the relation $\epsilon=\hbar \Omega_{\text {Rabi }}$ [22], with $\Omega_{\text {Rabi }} \stackrel{\text { def }}{=} e B_{\perp} /(2 m c)$. The quantities $e$ and $m$ are the charge and the mass of an electron. The quantity $c$ denotes the speed of light, while $B_{\perp}$ is the intensity of the magnetic field originating from the magnetic field components that are in the plane orthogonal to the quantization axis (that is, the $z$ axis). The energy gap $\hbar \omega_{0}$ is related to the Larmor angular frequency, $\hbar \omega_{0}=\hbar \Omega_{\text {Larmor }}$ [22], with $\Omega_{\text {Larmor }} \stackrel{\text { def }}{=} e B_{\|} /(m c)$. The quantity $B_{\|}$denotes the intensity of the magnetic field along the quantization axis. The Larmor frequency $v_{\text {Larmor }} \stackrel{\text { def }}{=} \Omega_{\text {Larmor }} /(2 \pi)$ of an electron in a magnetic field with $B_{\|}=1 \mathrm{~T}$ is:

$$
v_{\text {Larmor }}=\frac{1}{2 \pi}\left(\frac{e B_{\|}}{m c}\right)_{\mathrm{cgs}}=\frac{1}{2 \pi}\left(\frac{e B_{\|}}{m}\right)_{\mathrm{MKSA}} \simeq 28 \mathrm{GHz},
$$

where "cgs" and "MKSA" denote the physical unit system being used. Magnetic field intensities $B_{\|}$employed in MRI (magnetic resonance imaging) are typically in the range of 1-4 T. In the weak-driving regime [23], we consider a typical scenario where $B_{\|} \gg B_{\perp} \simeq 10^{-6} \mathrm{~T}=10^{-2} \mathrm{G}$ and $T_{A B}^{\text {(effective) }}=\frac{\pi}{2 \epsilon} \hbar \simeq 1.8 \times 10^{-5}$ sec. $\ll 1$. Observe that, since $T_{A B}^{\text {(effective) }} \propto 1 / B_{\perp}$, the condition $T_{A B}^{\text {(effective) }} \ll 1$ is satisfied by larger values of $B_{\perp}$ as well. Therefore, assumed to be in the short-time limit, from $\Delta E^{2}(t)$ in Equation (62) we Taylor expand $\Delta E(t)$ in the neighborhood of $t=0$. Then, the energy spread $\Delta E(t)$ reduces to:

$$
\Delta E(t)=\varepsilon\left[1+\frac{\omega_{0}^{2}}{2}\left(1+\frac{2 \omega}{\omega_{0}}\right) t^{2}\right]+\mathcal{O}\left(t^{4}\right),
$$

with $\mathcal{O}\left(t^{4}\right)$ denoting an infinitesimal quantity of order four or higher. From the condition that defines $T_{A B}^{(\text {ideal })} \mathrm{L}$

$$
\int_{0}^{T_{A B}^{(\mathrm{ideal})}} \Delta E(t) d t=\hbar \frac{\pi}{2}
$$

we obtain using Equation (64):

$$
T_{A B}^{\text {(effective) }}=\frac{\pi}{2 \epsilon} \hbar=\int_{0}^{T_{A B}^{(\text {ideal })}}\left[1+\frac{\omega_{0}^{2}}{2}\left(1+\frac{2 \omega}{\omega_{0}}\right) t^{2}\right] d t>\int_{0}^{T_{A B}^{(\text {ideal })}} d t=T_{A B}^{\text {(ideal) }} .
$$

The above inequality is justified by the positivity of the second addendum in the integrand in Equation (66). Therefore, $T_{A B}^{\text {(ideal) }}$ is upper bounded by the effective evolution time $T_{A B}^{(\text {effective) }}$. In particular, calculating the integral in Equation (66), $T_{A B}^{(\text {ideal })} \equiv \tilde{T}$ is implicitly defined by the relation:

$$
\frac{\pi}{2 \epsilon} \hbar=\tilde{T}+\frac{1}{3} a \tilde{T}^{3}
$$

with $a \stackrel{\text { def }}{=} \frac{\omega_{0}^{2}}{2}\left(1+\frac{2 \omega}{\omega_{0}}\right)>0$ and $[a]_{\text {MKSA }}=$ sec. $^{-2}$. Clearly, Equation (67) is a special case of Equation (33) and can only be numerically solved for $\tilde{T}$ once $\epsilon, \omega$, and $\omega_{0}$ are fixed. From 
Equation (66), we expect to obtain $\tilde{T}<T_{A B}^{\text {(effective) }}$ with the quantum motion occurring with geometric efficiency $\eta_{\mathrm{QM}}^{\text {(geometric) }}<1$.

The discussion of this time-dependent scenario illustrates the type of challenges that one may encounter when dealing with more realistic time-dependent scenarios.

\section{Concluding Remarks}

In this paper, we presented a simple proof of the fact that the minimum time $T_{A B}$ for the quantum evolution between two arbitrary states $|A\rangle$ and $|B\rangle$ equals $T_{A B}=\hbar \cos ^{-1}[|\langle A \mid B\rangle|] / \Delta E$ (see Equation (29)) with $\Delta E$ being the constant energy uncertainty of the system. This proof was performed in the absence of any geometrical arguments and closely followed the reasoning employed in Ref. [4] by Vaidman. Then, within the geometric framework of quantum evolutions based upon the geometry of the projective Hilbert space as developed by Anandan and Aharonov in Ref. [3], we discussed the roles played by either minimum-time or maximum-energy uncertainty concepts in defining a geometric efficiency measure (see $\eta_{\mathrm{QM}}^{\text {(geometric) }}$ in Equation (36)) of quantum evolutions between two arbitrary nonorthogonal quantum states. In particular, we provided a quantitative justification of the validity of the inequality $\eta_{\mathrm{QM}}^{\text {(geometric) }} \leq 1$ even when the system passed through nonorthogonal states (see Equation (49)). A schematic description of our main discussion points appears in Table 1.

While our investigation was performed in the spirit of the original Vaidman work, we additionally considered here a number of new modifications. Firstly, we extended the reasoning to unitary Schrödinger evolutions between quantum states that are not necessarily orthogonal. Secondly, we provided two explicit and alternative detailed proofs of the clever decomposition of $\hat{Q}|\psi\rangle$ in Equation (8) which plays a key role in the main proof itself. Thirdly, we emphasize its generalization to time-dependent Hamiltonian evolutions. Fourthly, and perhaps most importantly, we show the usefulness of the outcomes of the proof in upper bounding the geometric efficiency of quantum evolutions between two arbitrary states, either orthogonal or nonorthogonal. Lastly, we quantitatively present two illustrative examples discussing both time-independent and time-dependent quantum Hamiltonian evolutions in terms of minimum evolution time and geometric efficiency.

As a final remark, we point out that it would be interesting to further deepen our understanding of this geometric efficiency analysis to physical scenarios where the energy uncertainty $\Delta E$ is not constant in time. A partial list of scenarios that could be considered includes the $\operatorname{su}(2 ; \mathbb{C})$ time-dependent Hamiltonian evolutions used to describe distinct types of analog quantum search schemes viewed as driving strategies in Ref. [24] and, in addition, the time-dependent Hamiltonian describing the resonance phenomenon in a two-state quantum system used to construct quantum search algorithms by Wilczek and collaborators in Ref. [25] without limiting the analysis to the short-time limit of the nearresonance regime. We hope to address these more applied investigations in future efforts.

Author Contributions: Conceptualization, C.C.; Writing-original draft, C.C.; Writing-review and editing, C.C. and P.M.A.; Supervision, P.M.A. Both authors have read and agreed to the published version of the manuscript.

Funding: This research received no external funding.

Institutional Review Board Statement: Not applicable.

Informed Consent Statement: Not applicable.

Data Availability Statement: Not applicable.

Acknowledgments: C.C. is grateful to the United States Air Force Research Laboratory (AFRL) Summer Faculty Fellowship Program for providing support for this work. P.M.A. acknowledges support from the Air Force Office of Scientific Research (AFOSR). Any opinions, findings and conclusions or recommendations expressed in this material are those of the author(s) and do not necessarily reflect the views of the Air Force Research Laboratory (AFRL). 
Conflicts of Interest: The authors declare no conflict of interest.

\section{Appendix A. Alternative Derivation of Equation (8)}

In this Appendix, we provide an alternative derivation of Equation (8). Let us assume that the full Hilbert space $\mathcal{H}$ has an orthogonal decomposition given by $\mathcal{H} \stackrel{\text { def }}{=} \mathcal{H}_{\psi} \oplus \mathcal{H}_{\psi_{\perp}}$. Therefore, the identity operator $\hat{1}$ on $\mathcal{H}$ can be decomposed in terms of orthogonal projector operators as $\hat{1} \stackrel{\text { def }}{=} P_{|\psi\rangle}+P_{\left|\psi_{\perp}\right\rangle}$ with $P_{|\psi\rangle}$ and $P_{\left|\psi_{\perp}\right\rangle}$ given by $|\psi\rangle\langle\psi|$ and $\left|\psi_{\perp}\right\rangle\left\langle\psi_{\perp}\right|$, respectively. Then, for any operator $\hat{Q}$, the state $\hat{Q}|\psi\rangle$ can be recast as

$$
\begin{aligned}
\hat{Q}|\psi\rangle & =\hat{1} \hat{Q}|\psi\rangle \\
& =\left(P_{|\psi\rangle}+P_{\left|\psi_{\perp}\right\rangle}\right) \hat{Q}|\psi\rangle \\
& =\langle\psi|\hat{Q}| \psi\rangle|\psi\rangle+P_{\left|\psi_{\perp}\right\rangle} \hat{Q}|\psi\rangle,
\end{aligned}
$$

that is, setting $\langle\hat{Q}\rangle \stackrel{\text { def }}{=}\langle\psi|\hat{Q}| \psi\rangle$ :

$$
\hat{Q}|\psi\rangle=\langle\hat{Q}\rangle|\psi\rangle+\left[\left\langle\psi\left|\left(P_{\left|\psi_{\perp}\right\rangle} \hat{Q}\right)^{\dagger}\left(P_{\left|\psi_{\perp}\right\rangle} \hat{Q}\right)\right| \psi\right\rangle\right]^{1 / 2} \frac{P_{\left|\psi_{\perp}\right\rangle} \hat{Q}|\psi\rangle}{\left[\left\langle\psi\left|\left(P_{\left|\psi_{\perp}\right\rangle} \hat{Q}\right)^{\dagger}\left(P_{\left|\psi_{\perp}\right\rangle} \hat{Q}\right)\right| \psi\right\rangle\right]^{1 / 2}}
$$

Assuming that $\hat{Q}$ is an Hermitian operator, it happens that the amplitude $\left\langle\psi\left|\left(P_{\left|\psi_{\perp}\right\rangle} \hat{Q}\right)^{\dagger}\left(P_{\left|\psi_{\perp}\right\rangle} \hat{Q}\right)\right| \psi\right\rangle$ equals the dispersion $\Delta Q^{2}$ of the operator $\hat{Q}$ defined as $\Delta Q^{2} \stackrel{\text { def }}{=}\left\langle\psi\left|\hat{Q}^{2}\right| \psi\right\rangle-\langle\psi|\hat{Q}| \psi\rangle^{2}$. Therefore, Equation (A2) becomes:

$$
\hat{Q}|\psi\rangle=\langle\hat{Q}\rangle|\psi\rangle+\Delta Q\left|\psi_{\perp}\right\rangle,
$$

with the unit vector $\left|\psi_{\perp}\right\rangle$ in Equations (A3) given by

$$
\left|\psi_{\perp}\right\rangle \stackrel{\text { def }}{=} \frac{P_{\left|\psi_{\perp}\right\rangle} \hat{Q}|\psi\rangle}{\Delta Q}=\frac{\hat{Q}|\psi\rangle-\langle\hat{Q}\rangle|\psi\rangle}{\Delta Q} .
$$

The expression in Equation (A4) explicitly exhibits the orthonormal nature of $|\psi\rangle$ and $\left|\psi_{\perp}\right\rangle$, namely $\left\langle\psi_{\perp} \mid \psi_{\perp}\right\rangle=1$ and $\left\langle\psi \mid \psi_{\perp}\right\rangle=0$.

Equations (A3) and (A4) conclude our alternative proof of the simple, yet important, formula in Equation (8).

\section{References}

1. Mandelstam, L.; Tamm, I. The uncertainty relation between energy and time in non-relativistic quantum mechanics. J. Phys. 1945, $9,249$.

2. Fleming, G.N. A unitarity bound on the evolution of nonstationary states. Nuovo Cim. 1973, A16, 232. [CrossRef]

3. Anandan, J.; Aharonov, Y. Geometry of quantum evolution. Phys. Rev. Lett. 1990, 65, 1697. [CrossRef] [PubMed]

4. Vaidman, L. Minimum time for the evolution to an orthogonal quantum state. Am. J. Phys. 1992, 60, 182. [CrossRef]

5. Margolus, N.; Levitin, L.B. The maximum speed of dynamical evolution. Physica 1998, D120, 188.

6. Levitin, L.B.; Toffoli, T. Fundamental limit on the rate of quantum dynamics: The unified bound is tight. Phys. Rev. Lett. 2009, 103, 160502. [CrossRef] [PubMed]

7. Svozil, K.; Levitin, L.B.; Toffoli, T.; Walton, Z. Maximum speed of quantum gate operation. Int. J. Theor. Phys. 2005, 44, 965. [CrossRef]

8. Giovannetti, V.; Lloyd, S.; Maccone, L. Quantum limits to dynamical evolution. Phys. Rev. 2003, A67, 052109. [CrossRef]

9. Zander, C.; Plastino, A.R.; Plastino, A.; Casas, M. Entanglement and the speed of evolution of multipartite quantum systems. J. Phys. 2007, A40, 2861.

10. Frey, M.R. Quantum speed limits-primer, perspectives, and potential future directions, Quantum Inf. Process. 2016, 15, 3919. [CrossRef]

11. Merzbacker, E. Quantum Mechanics; John Wiley \& Sons, Inc.: Hoboken, NJ, USA, 1998. 
12. Aharonov, Y.; Vaidman, L. Properties of a quantum system during the time interval between two measurements. Phys. Rev. 1990, $A 41,11$. [CrossRef]

13. Bender, C.M.; Brody, D.C.; Jones, H.F.; Meister, B.K. Faster than Hermitian quantum mechanics. Phys. Rev. Lett. 2007, 98, 040403. [CrossRef] [PubMed]

14. Cafaro, C.; Ray, S.; Alsing, P.M. Geometric aspects of analog quantum search evolutions. Phys. Rev. 2020, A102, 052607. [CrossRef]

15. Wootters, W.K. Statistical distance and Hilbert space. Phys. Rev. 1981, D23, 357. [CrossRef]

16. Peres, A. Quantum Theory: Concepts and Methods; Kluwer Academic Publishers: New York, NY, USA, 1995.

17. Bengtsson, I.; Zyczkowski, K. Geometry of Quantum States; Cambridge University Press: Cambridge, UK, 2017

18. Cafaro, C.; Felice, D.; Alsing, P.M. Quantum Groverian geodesic paths with gravitational and thermal analogies. Eur. Phys. J. Plus 2020, 135, 900. [CrossRef]

19. Mostafazadeh, A. On Hamiltonians generating optimal-speed evolutions. Phys. Rev. 2009, A79, 014101. [CrossRef]

20. Laba, H.P.; Tkachuk, V.M. Geometric characteristics of quantum evolution: Curvature and torsion. Condens. Matter Phys. 2017, 20, 1. [CrossRef]

21. Scully, M.O.; Zubairy, M.S. Quantum Optics; Cambridge University Press: Cambridge, UK, 1994

22. Sakurai, J. J. Modern Quantum Mechanics; Addison-Wesley Publishing Company, Inc.: Boston, MA, USA, 1994.

23. Shim, J.H.; Lee, S.-J.; Yu, K.-K.; Hwang, S.-M.; Kim, K. Strong pulsed excitations using circularly polarized fields for ultra-low field NMR. J. Magn. Reson. 2014, 239, 87. [CrossRef] [PubMed]

24. Cafaro, C.; Gassner, S.; Alsing, P.M. Information geometric perspective on off-resonance effects in driven two-level quantum systems. Quantum Rep. 2020, 2, 166-188. [CrossRef]

25. Wilczek, F.; Hu, H.-Y.; Wu, B. Resonant quantum search with monitor qubits. Chin. Phys. Lett. 2020, 37, 050304. [CrossRef] 